jz-2021-03688f.R1

Name: Peer Review Information for "Activated Triplet Exciton Release for Highly Efficient RoomTemperature Phosphorescence Based on S, N Doped Polymeric Carbon Nitride"

First Round of Reviewer Comments

Reviewer: 1

Comments to the Author

In this manuscript, we report a strategy to promote the efficient phosphorescence to activate triplet exciton release by introduction of $\mathrm{S}$ and $\mathrm{N}$ elements. The introduction of $\mathrm{C}=\mathrm{S}$ and $\mathrm{C} \equiv \mathrm{N}$ groups in S, N-PCN networks could boost the intersystem crossing (ISC), leading to the small singlet-triplet energy $(\triangle \mathrm{EST})$ up to more triplet exciton generation. However, the crucial evidence was not provided to prove innovation point. In addition, the accuracy of the presentation and grammar should be carefully checked. As such, I recommend reject. More detailed comments are listed below:

1. The first occurrence of abbreviations should be pointed out, such as PQY, et. al.

2. In SI, the picture is not quoted correctly (Figure S3). In Figure S4 and Table S3, "Figure S4. FL emission (blue line) and corresponding phosphorescence emmision (orange line) spectra of the CDs0.", what does the CDs represent?

3. The preparation process of S, N-PCNO is unclear. Please provide the detailed preparation process of S, N-PCNO.

4. The last paragraph of page 10, how is that proof that producing more triplet state excitons by XPS and FT-IR analysis of S, NPCN? The detailed explanation should be provided to proof "activated triplet exciton release".

Reviewer: 2

Comments to the Author

The authors demonstrated activated triplet exciton release for highly efficient room temperature phosphorescence based on S, N doped polymeric carbon nitride. The experiments and results are interesting and new enough for The Journal of Physical Chemistry Letters. The overall quality of presented data and analysis is acceptable. I would like to recommend publication. However, the manuscript is not suitable for publication in the present form. Several confusing parts need to be clarified or corrected.

1. The authors described "the large singlet-triplet energy $(\Delta \mathrm{EST}=0.462 \mathrm{eV})$ doping $\mathrm{N}$ or $\mathrm{O}$ elements suffers from low PQY". It is required that related evidence to explain how singlettriplet energy $(\triangle E S T)$ influences phosphorescent performance. 
2. A series of PCN materials has been synthesized in the teflon by the authors at the low temperturature $\left(<300^{\circ} \mathrm{C}\right)$. Different from that, there is the traditional synthesis of carbon nitride in the tube or muffle furnace at above relatively high temperature $\left(400-600^{\circ} \mathrm{C}\right)$. Please show the significance of $\mathrm{S}, \mathrm{N}-\mathrm{PCN}$ in the teflon.

3. It is recommended that the authors standardize the format of the figures in the manuscript as well as the font size especially for Figure 1. Figure 1e XRD spectrum of N-PCN overlaps with the $x$-axis, suggested modification.

4. It will be better to revise the whole manuscript carefully and tried to avoid any grammar or syntax errors, such as "The origin of the RTP can be assigned that the $\mathrm{C}=\mathrm{N} / \mathrm{C}=\mathrm{O}$ groups on the surface of $\mathrm{PCN}$... groups such as $-\mathrm{NH} 2,-\mathrm{OH},-\mathrm{C}=\mathrm{O},-\mathrm{NH}$ and etc.". $\urcorner$

5. The reference 1 needs to be completed in the text.

Author's Response to Peer Review Comments:

Dear prof. Benjamin J. Schwartz and reviewers

Thank you for your letter and the reviewers' comments concerning our manuscript (ID. jz-202103688f Title: Activated Triplet Exciton Release for Highly Efficient Room Temperature Phosphorescence Based on S, N Doped Polymeric Carbon Nitride) and give us a chance to resubmit our revised manuscript. In response to these comments, we have checked our manuscript carefully and made a corresponding revision. We believe that we have addressed all the comments raised by the reviewers.

Following the kind suggestions from referees and editor, additional experiments have been carried out, and corresponding discussions were provided in the revised manuscript. Revisions in the text are shown using red highlight, and strikethrough font for deletions. Moreover, whole manuscript and supplement information have been revised carefully to avoid any grammar or syntax error. We believe that the paper will draw attention of peer researchers in the same field, and the contribution of this study warrants its publication in the Journal of Physical Chemistry Letters.

It is our great pleasure to submit the revised version of manuscript, response letter, and the change list to the reviewers. Additionally, more detailed experiments and analyses have been given in supplementary information. If you have further requirement, please do not hesitate to let me know.

Looking forward to hearing from you.

Thanks for your consideration. 
Shichen Su

\section{Reply to the Reviewers}

We feel grateful to the kind advice on our manuscript which has been submitted to the Journal of Physical Chemistry Letters (ID. jz-2021-03688f). In response to these comments, we have carried out further experimentation and theory analysis, and made a corresponding revision. We hope these are acceptable to allow the manuscript to be published.

The corresponding amendments to meet the referees' concerns have been marked in the revised draft. Our replies to the reviewers' suggestions and the corresponding changes are listed below.

The following are details of our response to editor and reviewers' comments:

\section{Editor:}

We also require a graphic for the "Table of Contents" with all submissions. We ask that you include a graphic immediately after the Abstract under the header "TOC Graphic."

Response: Following the editor's kind suggestion, we added a graphic after the Abstract under the header.

\section{Reviewer 1:}

Recommendation: This paper is not recommended because it does not provide new physical insights.

In this manuscript, we report a strategy to promote the efficient phosphorescence to activate triplet exciton release by introduction of $\mathrm{S}$ and $\mathrm{N}$ elements. The introduction of $\mathrm{C}=\mathrm{S}$ and $\mathrm{C} \equiv \mathrm{N}$ groups in S, N-PCN networks could boost the intersystem crossing (ISC), leading to the small singlet-triplet energy ( $\left.\Delta \mathrm{E}_{\mathrm{ST}}\right)$ up to more triplet exciton generation. However, the crucial evidence was not provided to prove innovation point. 
Comment 1: The first occurrence of abbreviations should be pointed out, such as PQY, et. al.

Response: We really thank the reviewer for pointing out the issues we did not describe clearly in the original manuscript. The full descriptions of the abbreviations like PQY have been supplemented in the revised manuscript. Revised on Page 3, Line 14 in the revised manuscript.

Comment 2: In SI, the picture is not quoted correctly (Figure S3). In Figure S4 and Table S3, "Figure S4. FL emission (blue line) and corresponding phosphorescence emission (orange line) spectra of the CDsO.", what does the CDs represent?

Response: We thanks the reviewer for pointing out the typos in the original SI. The quoted issues were revised. Changed "Figure S3" to "Figure S4" in Page 7, Line 3 in the revised SI. Changed "Figure S4" to "Figure S5" in the next sentence.

Comment 3: The preparation process of S, N-PCNO is unclear. Please provide the detailed preparation process of S, N-PCNO.

Response: We appreciate for the reviewers' constructive and helpful suggestion earnestly. In the revised manuscript, the synthesis of $\mathrm{S}, \mathrm{N}-\mathrm{PCNO}$ was added into experimental section as follows: " $0.6 \mathrm{~g}$ thiourea were uniformly ground in a mortar for $5 \mathrm{~min}$, and then transferred to a beaker for heating at $260^{\circ} \mathrm{C}$ for $12 \mathrm{~h}$. Then take out the beaker, after it cool down to room temperature naturally. After drying the powder at $40^{\circ} \mathrm{C}$, continue grinding for sample characterization. The obvious difference from S, N-PCN, prepared S, N-PCNO is an open reaction environment."

Comment 4: The last paragraph of page 10, how is that proof that producing more triplet state excitons by XPS and FT-IR analysis of S, NPCN? The detailed explanation should be provided to proof "activated triplet exciton release".

Response: We thank you for your insightful comments and critique of our work. Here, we do quantitative analysis as complementary evidence (measured time-resolved fluorescence spectra of N-PCN). We deem that there is the current proof of valid relationship between $\mathrm{C}=\mathrm{S}$ and $\mathrm{C} \equiv \mathrm{N}$ groups and performance of room temperature phosphorescence. Some of the crucial evidence will be presented in supporting information.

First. Fast ISC as a crucial photophysical process is related to the small singlet-triplet energy $\left(\Delta \mathrm{E}_{S T}\right)$ up to more triplet exciton generation. 
Accordingly, the phosphorescence efficiency $\left(\Phi_{P}\right)$ and lifetime $\left(\tau_{P}\right)$ can be expressed as ${ }^{1-4}$ :

$$
\begin{aligned}
& \Phi_{P}=\Phi_{I S C}\left[1-\left(k_{n r}+k_{q}\right)\right] \tau_{P} \\
& \Phi_{I S C}=\frac{k_{I S C}}{k_{F}+k_{I C}+k_{I S C}} \\
& \tau_{P}=\frac{1}{k_{P}+k_{n r}+k_{q}}
\end{aligned}
$$

where $\Phi_{\text {ISC }}$ is the quantum yield of ISC, and k represents the transition rate related to each process: $\mathrm{k}_{\mathrm{F}}$ is the fluorescent decay rate, $\mathrm{k}_{\mathrm{IC}}$ the internal conversion (IC) rate from $\mathrm{S}_{1}$ to $\mathrm{S}_{0}$, $\mathrm{k}_{\mathrm{ISC}}$ the ISC rate from $S_{1}$ to $T_{n}, k_{p}$ the phosphorescent decay rate, $k_{n r}$ the triplet non-radiative decay rate and $\mathrm{k}_{\mathrm{q}}$ the overall quenching rate of $\mathrm{T}_{1}$ after integrating the concentration of quenchers. ISC is the critical process for populating triplet excitons and, thus, determines the upper limit of $\Phi_{\mathrm{P}}$ (Eq. (1)). According to Eq. (2), organic phosphors with an ISC rate ( $\mathrm{k}_{\text {IsC }}$ ) fast enough $\left(<10^{11}\right.$ $\left.\mathrm{s}^{-1}\right)$ to compete with the fluorescent decay rate $\left(\mathrm{k}_{\mathrm{f}}, \sim 10^{7}-10^{10} \mathrm{~s}^{-1}\right)$ and the internal conversion rate $\left(k_{1 C},<10^{11} \mathrm{~s}^{-1}\right)$ can have a high yield of ISC ( $\left.\Phi_{\mid S C}\right) \cdot k_{\mid S C}$ is an intrinsic feature of organic molecules and it mostly depends on the electronic configuration and energy levels, which can be expressed as ${ }^{5,6}$ :

$$
k_{I S C}=\frac{2 \pi}{\hbar}|\langle S|\widehat{H} \operatorname{soc}| T\rangle|^{2} \sqrt{\frac{\pi}{\lambda k_{B} T}} \exp \left[-\frac{\left(\Delta E_{S T}-\lambda\right)^{2}}{4 \lambda k_{B} T}\right]
$$

where $\langle S|\widehat{H} s o c| T\rangle$ is the spin-orbit coupling (SOC) matrix element between singlet and triplet states, $\hbar$ is the reduced Planck's constant, $\lambda$ is the total reorganization energy, $k_{B}$ is the Boltzmann constant, $T$ is the temperature and $\Delta \mathrm{E}_{S \mathrm{~T}}$ represents the energy gap between the involved singlet and triplet states at their equilibrium geometries. Based on Eq. (4), the principles can guide the design of systems with improved ISC and provide some examples of PCN materials to facilitate the ISC process. It is reported, from a mechanistic perspective, tuning of the molecular orbital configurations based on the El-Sayed rule, reductions of the energy gap $\triangle \mathrm{E}_{\text {ST }}$ could facilitate the process of ISC, which enables the production of more triplet excitons through energy transfer ${ }^{5}$. According to the FL and PH spectra of S, N-PCN and N-PCN, the energy gap between $\Delta \mathrm{E}_{\mathrm{ST}}$ could be quantitatively determined to be 0.31 and $0.59 \mathrm{eV}$, respectively. Hence, in this work, the process of ISC for S, N-PCN is related to the small singlettriplet energy $\left(\triangle \mathrm{E}_{S T}\right)$ up to more triplet exciton generation.

Second. The introduction of $\mathrm{C}=\mathrm{S}$ and $\mathrm{C} \equiv \mathrm{N}$ groups in $\mathrm{S}, \mathrm{N}-\mathrm{PCN}$ networks could boost the ISC. The element doping treatment is a common technique that was often adopted \& reported in room temperature phosphorescence. SOC is the dominant driving force for ISC and can be greatly enhanced by tuning the electronic configurations of the involved singlet and triplet states. According to the El-Sayed rule, some aromatic compounds have weak SOC and slow $\mathrm{k}_{\mathrm{ISC}}$ due to slow transitions between ${ }^{1}\left(\pi-\pi^{*}\right)$ and ${ }^{3}\left(\pi-\pi^{*}\right)$. Through molecular engineering, carbonyl and groups containing other heteroatoms $\mathrm{O}, \mathrm{N}, \mathrm{S}$, and $\mathrm{P}$ with abundant lone pair electrons can be readily introduced ${ }^{7}$. Further, the introduction of $\mathrm{C}=\mathrm{S}$ and $\mathrm{C} \equiv \mathrm{N}$ groups including the lone pair 
electrons in S, N-PCN networks could be found by XPS and FT-IR data. Notably, the origin of phosphorescence of S, N-PCN is attributed to $n-\pi^{*}$ transition through the UV-visible absorption spectra. There is the obvious increased absorption of S, N-PCN compared N-PCN at the range from 250 to $700 \mathrm{~nm}$ to conclude $n-\pi^{*}$ transition from $C=S$ and $C \equiv N$ groups, corresponding to the smaller $\triangle \mathrm{E}_{S T}$ for $\mathrm{S}, \mathrm{N}-\mathrm{PCN}$. In addition, $\mathrm{S}, \mathrm{N}-\mathrm{PCN}$ exists prominent phosphorescent contrast to $\mathrm{S}$, $\mathrm{N}$-PCNO due to higher content of related $\mathrm{C}=\mathrm{S}$ and $\mathrm{C} \equiv \mathrm{N}$ groups from XRD, FT-IR, and XPS data. According to the corresponding trends between fine structure and phosphorescent spectra, we could infer the introduction of $\mathrm{C}=\mathrm{S}$ and $\mathrm{C} \equiv \mathrm{N}$ groups in $\mathrm{S}, \mathrm{N}-\mathrm{PCN}$ networks could boost the ISC process.

Third. We do quantitative analysis according to added time-resolved fluorescence spectra of $\mathrm{N}$ PCN for activated triplet exciton release ${ }^{8}$ :

$$
\begin{aligned}
k_{I S C} & =\frac{\Phi_{P}}{\tau_{F}} \\
k_{r}^{P} & =\frac{\Phi_{P}}{\tau_{P}}
\end{aligned}
$$

Where $\tau_{P}$ is phosphorescence lifetime, $\tau_{F}$ is fluorescence lifetime, $k_{r}^{P}$ is the triplet radiative decay rate. Figure $\mathrm{S} 7$ is revised and added time-resolved fluorescence spectrum of N-PCN:

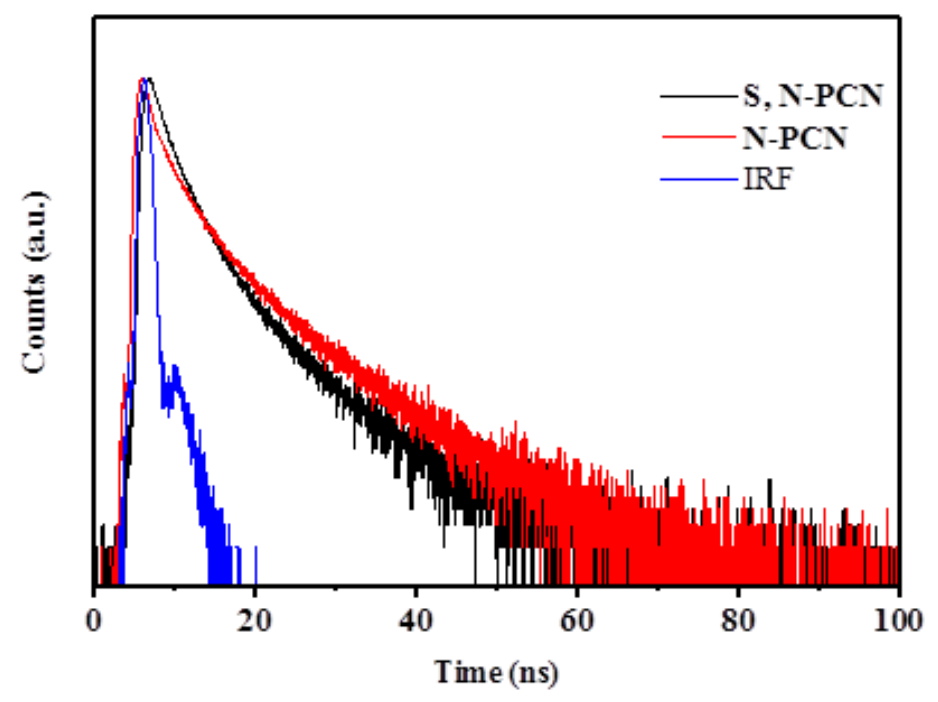

Figure S7

\begin{tabular}{|c|c|c|c|c|c|}
\hline Materials & $\begin{array}{l}\Phi_{P} \\
(\%)\end{array}$ & $\begin{array}{c}\boldsymbol{\tau}_{\boldsymbol{F}} \\
\text { (ns) }\end{array}$ & $\begin{array}{l}\boldsymbol{\tau}_{\boldsymbol{P}} \\
\text { (s) }\end{array}$ & $\begin{array}{c}\boldsymbol{k}_{I S C} \\
\left(\mathrm{~s}^{-1}\right)(5)\end{array}$ & $\begin{array}{c}\boldsymbol{k}_{\boldsymbol{r}}^{\boldsymbol{P}} \\
\left(\mathrm{s}^{-1}\right)(6)\end{array}$ \\
\hline S, N-PCN & 4.15 & 4.69 & 0.29 & $8.8 \times 10^{6}$ & $1.4 \times 10^{-1}$ \\
\hline $\mathrm{N}-\mathrm{PCN}$ & 0.41 & 5.58 & 0.74 & $7.2 \times 10^{5}$ & $5.5 \times 10^{-3}$ \\
\hline
\end{tabular}

Table S5. Dynamic photophysical parameters of S, N-PCN and N-PCN. 
The introduction of $\mathrm{C}=\mathrm{S}$ and $\mathrm{C} \equiv \mathrm{N}$ groups in $\mathrm{S}, \mathrm{N}-\mathrm{PCN}$ networks firmly promotes the ISC from singlet to triplet states and then leading to high efficient phosphorescence ( $P Q Y=4.15 \%$ ), which was further confirmed by a faster ISC rate, $k_{I S C},\left(8.8 \times 10^{6} \mathrm{~s}^{-1}\right)$ for S, N-PCN than those for N-PCN $\left(7.2 \times 10^{5} \mathrm{~s}^{-1}\right)($ Table S5). Furthermore, it was found that S, N-PCN showed a faster radiative transition, $k_{r}^{P},\left(1.4 \times 10^{-1} \mathrm{~s}^{-1}\right)$ and slower nonradiative decay rates $\left(5.5 \times 10^{-3} \mathrm{~s}^{-1}\right)$ compared with those for N-PCN (Table S5). Taking these results together, we reasoned that highly efficient phosphorescence was ascribed to suppressing the nonradiative transition and populating triplet excitons through the introduction of $\mathrm{C}=\mathrm{S}$ and $\mathrm{C} \equiv \mathrm{N}$ groups in $\mathrm{S}, \mathrm{N}-\mathrm{PCN}$ networks.

\section{Reviewer 2:}

Recommendation: This paper is publishable subject to minor revisions noted. Further review is not needed.

\section{Comments:}

The authors demonstrated activated triplet exciton release for highly efficient room temperature phosphorescence based on $\mathrm{S}, \mathrm{N}$ doped polymeric carbon nitride. The experiments and results are interesting and new enough for The Journal of Physical Chemistry Letters. The overall quality of presented data and analysis is acceptable. I would like to recommend publication. However, the manuscript is not suitable for publication in the present form. Several confusing parts need to be clarified or corrected.

Comment 1: The authors described "the large singlet-triplet energy $\left(\Delta E_{S T}=0.462 \mathrm{eV}\right)$ doping $N$ or $O$ elements suffers from low $P Q Y^{\prime \prime}$. It is required that related evidence to explain how singlettriplet energy $\left(\Delta E_{S T}\right)$ influences phosphorescent performance.

Response: We thank you for your insightful comments and critique of our work. The small singlet-triplet energy $\left(\Delta \mathrm{E}_{S T}\right)$ is up to more triplet exciton generation. Tang's groups reviewed the theoretical evidence: "tuning of the molecular orbital configurations based on the El-Sayed rule, the heavy-atom effect, hyperfine coupling, reductions of the energy gap $\Delta \mathrm{E}_{\mathrm{s} T}$ by bridging charge-transfer states and aggregation-facilitated ISC, which enables the production of more triplet excitons through energy transfer." ${ }^{5}$. In addition, Huang's groups demonstrate that the organic afterglow can be enhanced dramatically by thermally activated processes to release the excitons on the stabilized triplet state $\left(T_{1}{ }^{*}\right)$ to the lowest triplet state $\left(T_{1}\right)$, meaning small singlet-triplet splitting energy, and to the singlet excited state $\left(\mathrm{S}_{1}\right)$ for spin-allowed emission ${ }^{7}$. Hence, the small singlet-triplet energy $\left(\Delta \mathrm{E}_{S T}\right)$ is up to more triplet exciton generation, leading to high phosphorescence quantum yield. 
Comment 2: A series of PCN materials has been synthesized in the teflon by the authors at the low temperature $\left(<300^{\circ} \mathrm{C}\right)$. Different from that, there is the traditional synthesis of carbon nitride in the tube or muffle furnace at above relatively high temperature $\left(400-600^{\circ} \mathrm{C}\right)$. Please show the significance of S, N-PCN in the teflon.

Response: We found polymeric carbon nitride heating in the teflon (phosphorescent lifetime > $200 \mathrm{~ms}$ ) has even more distinguished performance in the unsealed glass (<100 ms). This is a universal strategy so that polymeric carbon nitride could be synthesized with high performance in the teflon ${ }^{8}$. There is a preliminary exploration for the mechanism of phosphorescent materials in the closed reactors, which is of value to understand the relationship between the structure and function of phosphorescent materials.

Comment 3: It is recommended that the authors standardize the format of the figures in the manuscript as well as the font size especially for Figure 1. Figure 1e XRD spectrum of N-PCN overlaps with the $x$-axis, suggested modification.

Response: We really thank the reviewer for pointing out the issues. According to the reviewer's comment, XRD spectrum of N-PCN were revised Figure 1e:

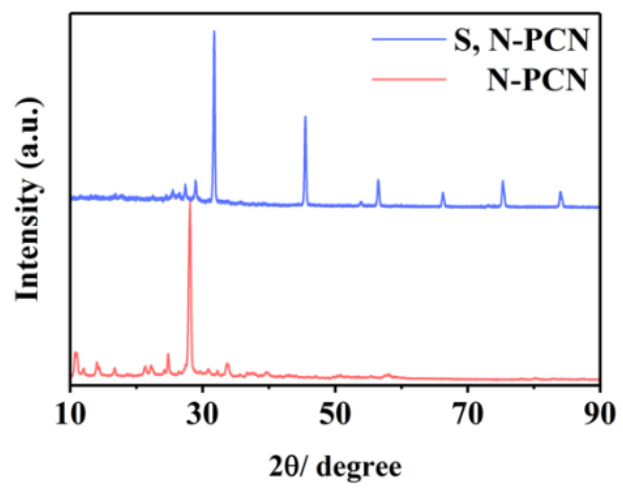

Figure 1e

Comment 4: It will be better to revise the whole manuscript carefully and tried to avoid any grammar or syntax errors, such as "The origin of the RTP can be assigned that the $C=N / C=O$ groups on the surface of $\mathrm{PCN}$... groups such as $-\mathrm{NH}_{2},-\mathrm{OH},-\mathrm{C}=\mathrm{O},-\mathrm{NH}$ and etc.".

Response: We are very sorry for the mistakes in this manuscript and inconvenience they caused in your reading. The manuscript has been thoroughly revised and edited, so we hope it can meet the journal's standard. Thanks so much for your useful comments. The sentence changed to "The origin of the RTP can be assigned that the $\mathrm{C}=\mathrm{N} / \mathrm{C}=\mathrm{O}$ groups on the surface of $\mathrm{PCN}$, 
which is fixed in the PVA matrix, provide structural rigidity, favoring ISC and stabilizing the triplet state. On the other hand, it was reported that RTP also happened on matrix-free PCN, which shows $\mathrm{H}$-bonded supramolecular aggregate and a large variety of functional groups such as $-\mathrm{NH}_{2},-\mathrm{OH},-\mathrm{C}=\mathrm{O}$, and $-\mathrm{NH}$ etc. on it."

\section{Comment 5: The reference 1 needs to be completed in the text."}

Response: We thank the reviewers for pointing out the reference format problem. The references are added and reordered.

\section{Chang list in manuscript:}

1. Added Table of Contents after the Abstract under the header.

2. Revised the figure $1 \mathrm{e}$ in the revised manuscript.

3. Changed "PQY" to "phosphorescence quantum yield (PQY)" in Page 3, Line 12;

4. Revised and reordered the references in the revised manuscript.

5. Revised the whole manuscript carefully and tried to avoid any grammar or syntax error:

Deleted "the" in Page 1, Line 14;

Removed spaces between numerals and units " $0.41 \%$ " in Page 1, Line 18;

Deleted the sentence: "Meanwhile, S, N-PCN has wide strong absorption reaching $700 \mathrm{~nm}$, resulting in readily visible-light-excitation." in Page 2, Line 2;

Deleted the sentence: "The room temperature phosphorescence based on S, N doped polymeric carbon nitride represents an important concept advance in designing high-efficient organic phosphorescence materials through facilitating generation of triplet excitons." in Page 2, Line 3;

Changed "material" to "materials" in Page 2, Line 10;

Changed "as a kind of photoluminescent materials" to "unlike fluorescence materials" in Page 2, Line 10 ;

Changed "Moreover" to "Additionally" in Page 2, Line 14;

Moved this paragraph "due to tunable color emission in organic RTP materials derived from various degrees of aggregation and polymerization" to the end of the sentence in Page 2, Line 15-17;

Changed "is" to "are" in Page 2, Line 17;

Changed "system" to "systems" in Page 2, Line 18;

Changed "researches" to "research" in Page 3, Line 5;

Changed "have" to "has" in Page 3, Line 6;

Revised the sentence to "The origin of the RTP can be assigned that the $\mathrm{C}=\mathrm{N} / \mathrm{C}=\mathrm{O}$ groups on the surface of PCN, which is fixed in the PVA matrix, provide structural rigidity, favoring ISC and stabilizing the triplet state. On the other hand, it was reported that RTP also happened on matrix-free PCN, which shows H-bonded supramolecular aggregate and a large variety of functional groups such as $-\mathrm{NH} 2$, $-\mathrm{OH},-\mathrm{C}=\mathrm{O}$, and $-\mathrm{NH}$ etc. on it." in Page 3, Line 6-11;

Changed "using" to "employing" in Page 3, Line 16;

Removed spaces between numerals and units "3.45 \%" in Page 1, Line 20;

Changed "is" to "are" in Page 4, Line 2; 
Removed spaces between numerals and units " $0.41 \%$ " in Page 4, Line 8;

Changed "undergoes" to "undergo" in Page 5, Line 12;

Changed "characterized" to "identified" in Page 6, Line 3;

Deleted "to" between "indicating" and "various" in Page 6, Line 5;

Changed "exists" to "occurs" in Page 6, Line 11;

Changed "keep" to "maintain" in Page 6, Line 15;

Changed "wxere" to "were" in Page 6, Line 19;

Changed "was" to "are" in Page 7, Line 4;

Added "is" between "S, N-PCN" and "located" in Page 7, Line 6;

Added "is" between "S, N-PCN" and "located" in Page 7, Line 7;

Changed "band exists" to "bands exist" in Page 7, Line 17;

Changed "week" to "weak" in Page 9, Line 9;

Added "of" between "lack" and "thiourea" in Page 9, Line 9;

Changed "week" to "weak" in Page 9, Line 9;

Font size of "According" has been corrected in Page 9, Line 11;

Font size of "The" has been corrected in Page 9, Line 11;

Changed "yeild" to "yield" in Page 9, Line 15;

Changed "phosphorescence quantum yield (PQY)" to "PQY" in Page 9, Line 15;

Changed "produces" to "produce" in Page 9, Line 18;

Added "in" after "exists" in Page 11, Line 3;

Changed "The" to "the" in Page 11, Line 4;

Changed "concered" to "concentrated" in Page 11, Line 6;

Deleted "the" between "with" and "lifetime" in Page 11, Line 7;

Changed "In" to "As a" in Page 11, Line 10;

Changed "ploymer" to "polymer" in Page 11, Line 11;

Changed "characteritic" to "characteristic" in Page 11, Line 12;

Deleted "the" between "In" and "light" in Page 12, Line 4;

Removed spaces between numerals and units "0.41 \%" in Page 13, Line 4;

Changed "wide" to "broad" in Page 13, Line 7;

Changed "excited" to "stimulated" in Page 13, Line 8;

Added "to" between "Due" and "the" in Page 13, Line 8;

\section{Change list in the Supporting Information:}

1. In Page 2, Line 14-18, added the synthesis process of S, N-PCN0

2. Added the explain of facilitating ISC rule in Page 3-4.

3. Added the Fl decay spectrum N-PCN (Figure S7) in Page 8.

4. Changed "CDs0" to "S N-PCNO" in Page 6, Line 4;

5. Changed "Figure S3" to "Figure S4" in Page 7, Line 3 in the revised SI. Changed "Figure S4" to "Figure S5" in the next sentence.

6. Added supplementary explain: "The time-resolved fluorescence spectra of S, N-PCN and N-PCN were also fitted with a tri-exponential function with an average lifetime of 4.69 and $5.58 \mathrm{~ns}$, respectively (Figure $\mathrm{S} 7$ ). The introduction of $\mathrm{C}=\mathrm{S}$ and $\mathrm{C} \equiv \mathrm{N}$ groups in $\mathrm{S}, \mathrm{N}-\mathrm{PCN}$ networks firmly promotes the ISC from singlet to triplet states and then leading to highly efficient phosphorescence, which was further 
confirmed by a faster ISC rate, $k_{I S C},\left(8.8 \times 10^{6} \mathrm{~s}^{-1}\right)$ for $\mathrm{S}, \mathrm{N}-\mathrm{PCN}$ than those for N-PCN $\left(7.2 \times 10^{5} \mathrm{~s}^{-1}\right)$ (Table S5). Furthermore, it was found that S, N-PCN showed a faster radiative transition, $k_{r}^{P},\left(1.4 \times 10^{-}\right.$ $\left.{ }^{1} \mathrm{~s}^{-1}\right)$ and slower nonradiative decay rates $\left(5.5 \times 10^{-3} \mathrm{~s}^{-1}\right)$ compared with those for N-PCN (Table S5)." in Page 9, Line 1-9.

7. Changed "CDs1, CDs0 and U-CDs" to "S, N-PCN, S, N-PCN0, and N-PCN" in Page 12.

8. Added supplementary data, $\mathrm{k}_{\mathrm{ISC}}$ and $\mathrm{k}_{\mathrm{r}}$ (Table S5) in Page 13.

9. Revised the whole SI carefully and tried to avoid any grammar or syntax error:

Changed "spectrum" to "spectra" in Page 4, Line 11;

Changed "spectrum" to "spectra" in Page 5, Line 7;

Changed "emmision" to "emission" in Page 6, Line 3;

Changed "spectra" to "spectrum" in Page 7, Line 3;

Changed "Fig. S6. Phosphorescence decay spectrum of S, N-PCN" to "Figure S6. (a) Phosphorescence and (b) phosphorescence decay spectra of S, N-PCN" in Page 7, Line 10;

Changed "Fig. S7. FL decay spectrum and IRF of S, N-PCN $(\lambda e x=405 \mathrm{~nm}, \lambda \mathrm{em}=480 \mathrm{~nm})$." to "Figure S7. Fl decay spectra and IRF of S, N-PCN $(\lambda e x=405 \mathrm{~nm}, \lambda \mathrm{em}=480 \mathrm{~nm})$ and N-PCN $(\lambda \mathrm{ex}=405 \mathrm{~nm}$, $\lambda \mathrm{em}=430 \mathrm{~nm}$ )" in Page 8, Line 3;

Changed "spectrum" to "spectra" in Page 9, Line 15;

Revised the font of annotation and added "S, N-PCN0" (Table S4) in Page 13.

\section{Reference}

(1) Hirata, S.; Totani, K.; Zhang, J.; Yamashita, T.; Kaji, H.; Marder, S. R.; Watanabe, T.; Adachi, C. Efficient Persistent Room Temperature Phosphorescence in Organic Amorphous Materials under Ambient Conditions. Adv. Funct. Mater. 2013, 23, 3386-3397.

(2) Ma, X.; Xu, C.; Wang, J.; Tian, H. Amorphous Pure Organic Polymers for Heavy-AtomFree Efficient Room-Temperature Phosphorescence Emission. Angew Chem Int Ed Engl 2018, 57, 10854-10858.

(3) Zhao, W.; Cheung, T. S.; Jiang, N.; Huang, W.; Lam, J. W. Y.; Zhang, X.; He, Z.; Tang, B. $Z$. Boosting the efficiency of organic persistent room-temperature phosphorescence by intramolecular triplet-triplet energy transfer. Nat Commun 2019, 10, 1595.

(4) Bian, L.; Shi, H.; Wang, X.; Ling, K.; Ma, H.; Li, M.; Cheng, Z.; Ma, C.; Cai, S.; Wu, Q.; Gan, N.; Xu, X.; An, Z.; Huang, W. Simultaneously Enhancing Efficiency and Lifetime of Ultralong Organic Phosphorescence Materials by Molecular Self-Assembly. J. Am. Chem. Soc. 2018, 140, 10734-10739.

(5) Zhao, W.; He, Z.; Tang, B. Z. Room-Temperature Phosphorescence from Organic Aggregates. Nat. Rev. Mater. 2020, 5, 869-885.

(6) Penfold, T. J.; Gindensperger, E.; Daniel, C.; Marian, C. M. Spin-Vibronic Mechanism for Intersystem Crossing. Chem. Rev. 2018, 118, 6975-7025.

(7) Jin, J.; Jiang, H.; Yang, Q.; Tang, L.; Tao, Y.; Li, Y.; Chen, R.; Zheng, C.; Fan, Q.; Zhang, K. Y.; Zhao, Q.; Huang, W. Thermally activated triplet exciton release for highly efficient tri-mode organic afterglow. Nat Commun 2020, 11, 842.

(8) Cheng, J.; Hu, Z.; Lv, K.; Wu, X.; Li, Q.; Li, Y.; Li, X.; Sun, J. Drastic promoting the visible photoreactivity of layered carbon nitride by polymerization of dicyandiamide at high 
pressure. Appl. Catal. B Environ. 2018, 232, 330-339.

\section{Jz-2021-03688f.R2}

Name: Peer Review Information for "Activated Triplet Exciton Release for Highly Efficient RoomTemperature Phosphorescence Based on S, N Doped Polymeric Carbon Nitride"

\section{Second Round of Reviewer Comments}

Reviewer: 1

\section{Comments to the Author}

In the revised manuscript, the authors answered my question clearly and accurately. I suggest the author be more rigorous about the format of the paper in the future. In addition, I think the PCN in the manuscript has similar synthetic method and properties with doped carbon dots. The authors should consider the accuracy of naming, and provide some structure information with PCN in the revised manuscript.

Author's Response to Peer Review Comments:

Dear editor and reviewers

Thank you for your letter and the reviewers' comments concerning our manuscript (ID. jz-2021$03688 \mathrm{f}$ Title: Activated Triplet Exciton Release for Highly Efficient Room Temperature Phosphorescence Based on S, N Doped Polymeric Carbon Nitride).

We have accepted some changes to the reviewer's comments and updated change list in revised manuscript R2 and the Supporting Information R2. Please find the new files in the attachment.

It is our great pleasure to submit response letter. If you have further requirement, please do not hesitate to let me know.

Looking forward to hearing from you.

Thanks for your consideration.

Shichen Su 


\section{Reply to Editor}

We feel grateful to the kind advice on our revised manuscript which has been submitted to the Journal of Physical Chemistry Letters (ID. jz-2021-03688f). In response to these comments, we have made a corresponding revision. We hope these are acceptable to allow the manuscript to be published.

The following are details of our response to editor and reviewers' comments:

\section{Reviewer:}

"In the revised manuscript, the authors answered my question clearly and accurately. I suggest the author be more rigorous about the format of the paper in the future. In addition, I think the PCN in the manuscript has similar synthetic method and properties with doped carbon dots. The authors should consider the accuracy of naming, and provide some structure information with PCN in the revised manuscript."

Response: We thank you for your insightful comments and critique of our work. We answer each of these questions one by one.

"In addition, I think the PCN in the manuscript has similar synthetic method and properties with doped carbon dots. The authors should consider the accuracy of naming."

Fortunately, we've also thought about your insightful comments before, and it isn't to be considered the naming of polymeric carbon nitride (PCN) need to be changed. Indeed, PCN has different fine structure from carbon dots, even though it exhibits some degree of similarity, including the same bottom-up method. Polymer carbon dots possess special "core-shell" nanostructures, consisting of carbon cores less than $20 \mathrm{~nm}$ with highly dehydrated crosslinking polymer frames or slight graphitization and shells of abundant functional groups/polymer chains ${ }^{1}$. But, the size from the TEM of S, N-PCN we measured shows larger than $100 \mathrm{~nm}$ (Figure $1 c$ in the manuscript). Further, it is found only a few of dispersed nanoparticles, when we tried our best to find traces of carbon dots form the TEM measurement (Figure R1), again. In addition, the presence of dispersed nanoparticles couldn't be proven by XRD data (Figure S1 in Supporting Information), because XRD of carbon dots usually exhibits a very wide peak, at around $22^{\circ 2,3}$. 


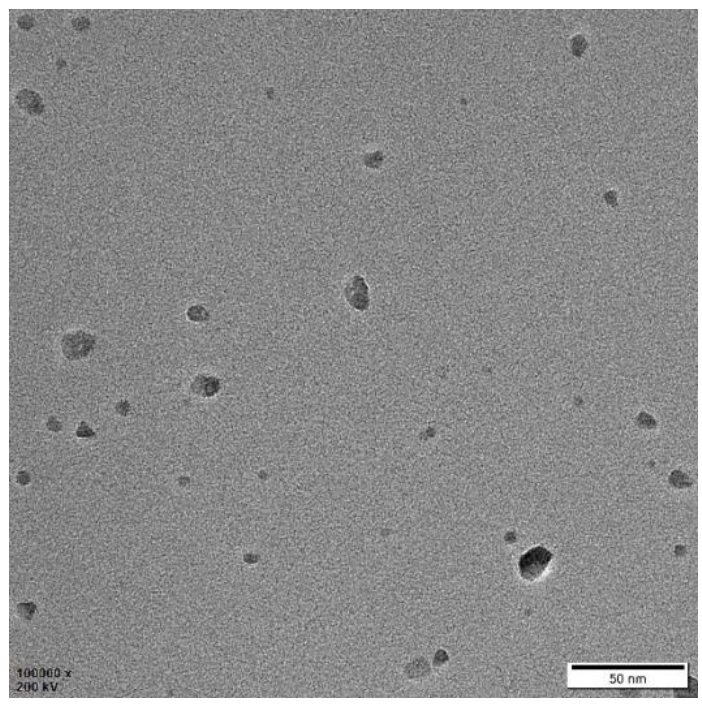

Figure R1. TEM image of S, N-PCN.

Only a few of carbon dots cannot form an energy transfer system (carbon dots is not in the matrix) with a polymer-like substance, even though a lot of experiments have found that the significant improvement of phosphorescence performance may be derived from the effect of infinitesimal dopants ${ }^{4,5}$.

\section{No correction.}

"The authors should provide some structure information with PCN in the revised manuscript."

We appreciate for the reviewers' constructive and helpful suggestion earnestly. We set out the reaction route and explained it: "Polymerization inevitably has some degrees in difference of size and crystal structure. In Figure S1(a) PCN shows a tiny peak at $27.4^{\circ}$, corresponding to the heptazine heterocyclic ring units ${ }^{6}$. It means the formation of polycrystalline compounds ( $B$ $\mathrm{C}_{3} \mathrm{~N}_{4}$ and $g-\mathrm{C}_{3} \mathrm{~N}_{4}$ ), but it is main composed of $B-\mathrm{C}_{3} \mathrm{~N}_{4}$. $B-\mathrm{C}_{3} \mathrm{~N}_{4}$ has simlar structure to $B-\mathrm{Si}_{3} \mathrm{~N}_{4}$, which is substituted for $\mathrm{Si}$ with $\mathrm{C}$ in the $\mathrm{B}-\mathrm{Si}_{3} \mathrm{~N}_{4}$ structure $^{7}$. Proposed possible reaction route of $\mathrm{S}, \mathrm{N}-\mathrm{PCN}$ from condensation of thiourea (Figure R2). Due to the incomplete reaction, intermediate products may be involved in the reaction, which leads to the presence of $\mathrm{S}$, and $\mathrm{N}$ elements ${ }^{8} . "$ 


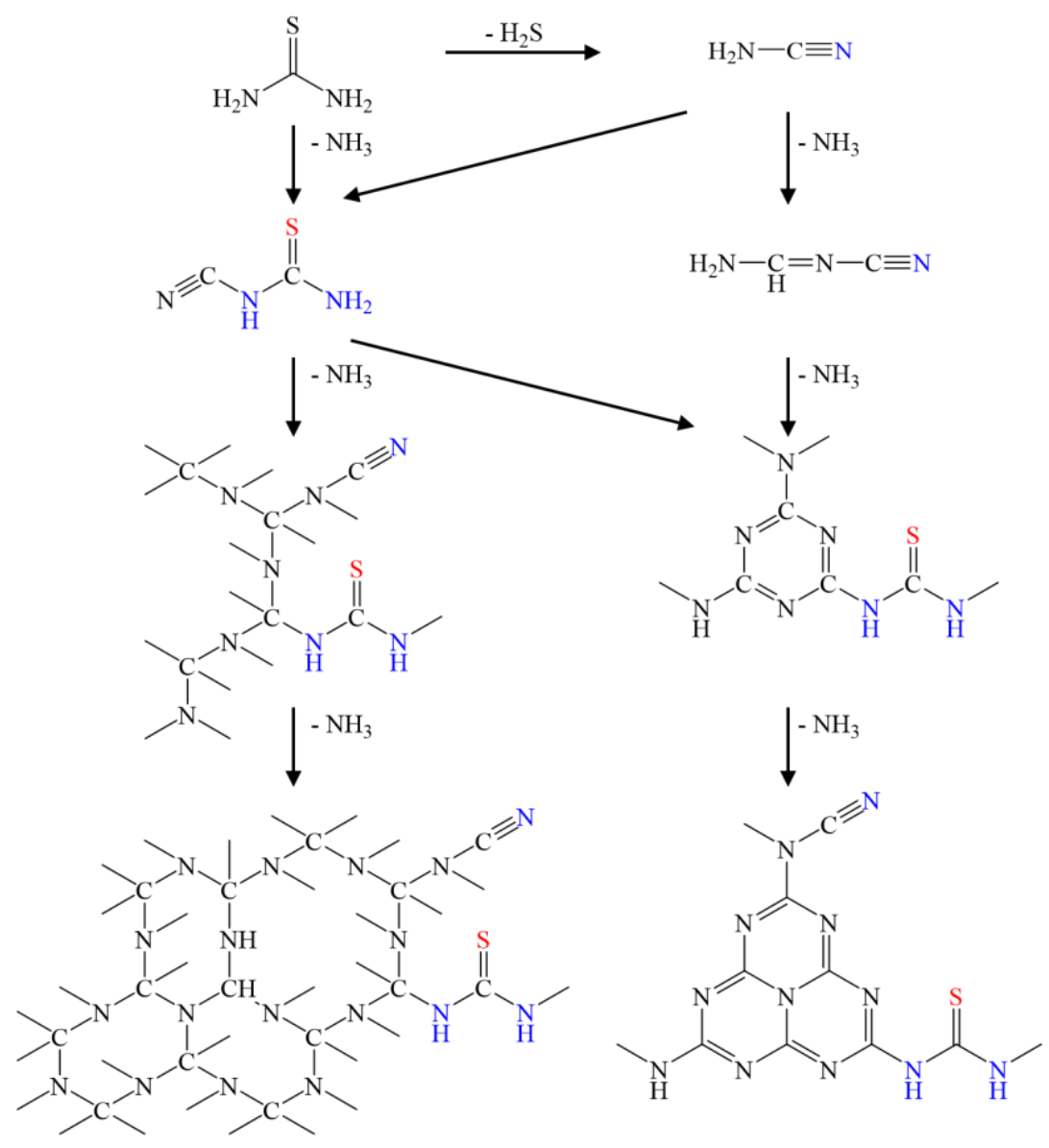

$\mathrm{S}$, N-polymeric carbon nitride $\left(\beta-\mathrm{C}_{3} \mathrm{~N}_{4}\right.$ (left) and $g-\mathrm{C}_{3} \mathrm{~N}_{4}$ (right) $)$

Figure R2. Possible reaction route of S, N-PCN from polymerization of thiourea.

We are sorry that it needs to be further explored the relationship between optical physical properties and structure due to limited experimental conditions. We will explore this issue in more detail in our next work.

Correction: We added possible reaction route of $\mathrm{S}, \mathrm{N}-\mathrm{PCN}$ for $\mathrm{B}-\mathrm{C}_{3} \mathrm{~N}_{4}$ in Figure $\mathrm{S} 1$ in the supporting information (Page 4-5) and the corresponding structure in TOC in revised manuscript (Page 1, Line 7).

\section{Change list in revised manuscript R2:}

6. Remove the colored text.

7. Revised the title case format.

8. Corrected Title, Full Author List, and Author affiliations.

9. Added and updated Table of Contents after the Abstract under the header in Page 2, Line 3.

10. Re-upload Figure 1; Substitute N-PCN data for S, N-PCN0 data (Figure 1g) in Page 5, Line 1.

11. References with more than 10 authors has listed the first 10 authors (Ref. 11). 


\section{Change list in the Supporting Information R2:}

10. Number the pages in the Supporting information have been added.

11. Remove the colored text.

12. Revised the title case format.

13. Corrected Title, Full Author List, and Author affiliations.

14. Changed "PQY" to "yield" in Page 2, Line 2.

15. Added Possible reaction path of $\mathrm{S}, \mathrm{N}-\mathrm{PCN}$ for $\beta-\mathrm{C}_{3} \mathrm{~N}_{4}$ in Figure $\mathrm{S} 1$ in the supporting information. Added supplementary explain: "Polymerization inculdes some degrees of size and crystal structure. In Figure S1(a) PCN shows a tiny peak at $27.4^{\circ}$, corresponding to the heptazine heterocyclic ring units. It means the formation of polycrystalline compounds $\left(\beta-\mathrm{C}_{3} \mathrm{~N}_{4}\right.$ and $\left.g-\mathrm{C}_{3} \mathrm{~N}_{4}\right)$, but it is main composed of $\beta-\mathrm{C}_{3} \mathrm{~N}_{4} . \beta-\mathrm{C}_{3} \mathrm{~N}_{4}$ has simlar structure to $\beta-\mathrm{Si}_{3} \mathrm{~N}_{4}$, which is substituted for $\mathrm{Si}$ with $\mathrm{C}$ in the $\beta-\mathrm{Si}_{3} \mathrm{~N}_{4}$ structure. In Figure S1(b), it shows the possible routes of reaction involving intermediates and final products. Due to the incomplete reaction, intermediate products may be involved in the reaction, which leads to the presence of special S, and N elements doped." in Page 4-5.

\section{Reference}

(1) Liu, J.; Li, R.; Yang, B. Carbon Dots: A New Type of Carbon-Based Nanomaterial with Wide Applications. ACS Central Science 2020, 6, 2179-2195.

(2) Wu, Z. L.; Liu, Z. X.; Yuana, Y. H. Carbon dots: materials, synthesis, properties and approaches to long-wavelength and multicolor emission. J. Mater. Chem. B 2017, 5, 37943809.

(3) Yuan, T.; Yuan, F.; Li, X.; Li, Y.; Fan, L.; Yang, S. Fluorescence-Phosphorescence Dual Emissive Carbon Nitride Quantum Dots Scores 25\% White Emission Efficiency Enabling Single-Component WLEDs. Chem. Sci. 2019, 10, 9801-9806.

(4) Kabe, R.; Adachi, C. Organic long persistent luminescence. Nature 2017, 550, 384-387.

(5) Wang, B. L.; Yu, Y.; Zhang, H.; Xuan, Y.; Chen, G.; Ma, W.; Li, J.; Yu, J. Carbon Dots in a matrix: energy-transfer-enhanced room-temperature red phosphorescence. Angew. Chem. Int. Ed. 2019, 58, 18443-18448.

(6) Zhang, G.; Zhang, J.; Zhang, M.; Wang, X. Polycondensation of thiourea into carbon nitride semiconductors as visible light photocatalysts. J. Mater. Chem. 2012, 22, 8083.

(7) Liu, A. Y.; Cohen, M. L. Structural properties and electronic structure of lowcompressibility materials: $\beta$-Si3N4and hypothetical $\beta$-C3N4. Phys. Rev. B 1990, 41, $10727-10734$.

(8) Li, Y.; Jin, S.; Xu, X.; Wang, H.; Zhang, X. Excitonic effects on photophysical processes of polymeric carbon nitride. J. Appl. Phys. 2020, 127, 